\title{
Optimisation De La Stabilisation Physique Du Lait De Coco En Utilisant La Gomme De Cajou (Anacardium occidentale L.) Comme Agent Stabilisant
}

\author{
Pégnonsienrè Lacina Soro
}

Université Nangui Abrogoua, UFR Sciences et Technologies des Aliments, Abidjan, Côte d'Ivoire

Naka Touré

Université Peleforo Gon Coulibaly,

UFR Sciences Biologiques, Korhogo, Côte d'Ivoire

Fankroma Martial Thierry Koné

Marcellin Konan Kouadio

Kouakou Martin Djè

Soumaila Dabonné

Université Nangui Abrogoua,

UFR Sciences et Technologies des Aliments, Abidjan, Côte d'Ivoire

Doi:10.19044/esj.2021.v17n7p227

Submitted: 08 November 2020

Accepted: 17 February 2021

Published: 28 February 2021
Copyright 2021 Author(s)

Under Creative Commons BY-NC-ND

4.0 OPEN ACCESS

Cite As:

Soro P.L., Touré N., Koné F.M.T., Kouadio M.K., Djè K.M. \& Dabonné S. (2021). Optimisation De La Stabilisation Physique Du Lait De Coco En Utilisant La Gomme De Cajou (Anacardium occidentale L.) Comme Agent Stabilisant. European Scientific Journal, ESJ, 17(7), 227. https://doi.org/10.19044/esj.2021.v17n7p227

\section{Résumé}

Le lait de coco est essentiellement une émulsion huile dans eau, stabilisée par les protéines naturelles et les phospholipides présents dans la pulpe. Cependant, le lait de coco reste physiquement instable et sujet à une séparation de phase. D'où la nécessité d'utiliser des agents stabilisants. Cette étude avait pour objectif de déterminer les conditions optimales de stabilisation du lait de coco en utilisant la gomme de cajou comme agent stabilisant, par la méthodologie des surfaces de réponse utilisant un plan factoriel complet 32. Ainsi, les effets de la concentration en gomme de cajou et du ratio pulpes/eau sur les paramètres physico-chimiques du lait de coco tels que l'indice de crémage, l'indice de taille des gouttelettes, la viscosité et 
le taux de perte de turbidité ont été évalués. Les résultats de l'analyse ont indiqué que les conditions optimales étaient de $1,50 \%$ pour la concentration en gomme de cajou et de $1 / 1(\mathrm{~g} / \mathrm{mL})$ pour le ratio pulpes/eau. Les réponses optimales enregistrées ont été de 60,239\%;0,716;0,076 mPa.s et de 0,824 $\AA / \mathrm{jr}$ respectivement pour l'indice de crémage, l'indice de taille des gouttelettes, la viscosité et le taux de perte de turbidité. Par ailleurs, aucune différence significative $(\mathrm{p}>0,05)$ n'a été observé entre les valeurs expérimentales et les valeurs prédites. Les différents modèles développés pour prédire les différentes caractéristiques du lait de coco étaient très significatifs, avec des coefficients de détermination élevés $(>0,95)$ et des manques d'ajustement non significatifs ( $p>0,05)$. La gomme de cajou porterait donc un fort potentiel dans la stabilisation des émulsions.

Mots clés : Lait De Coco, Stabilisation, Gomme De Cajou, Optimisation, Méthodologie De Surface De Réponse

\title{
Optimization Physical Stabilization of Coconut-Milk Using Cashew Tree (Anacardium Occidentale) Gum as Stabilizer
}

\author{
Pégnonsienrè Lacina Soro \\ Université Nangui Abrogoua, \\ UFR Sciences et Technologies des Aliments, Abidjan, Côte d'Ivoire \\ Naka Touré \\ Université Peleforo Gon Coulibaly, \\ UFR Sciences Biologiques, Korhogo, Côte d'Ivoire \\ Fankroma Martial Thierry Koné \\ Marcellin Konan Kouadio \\ Kouakou Martin Djè \\ Soumaila Dabonné \\ Université Nangui Abrogoua, \\ UFR Sciences et Technologies des Aliments, Abidjan, Côte d'Ivoire
}

\begin{abstract}
Coconut milk is essentially an oil-in-water emulsion, stabilized by natural proteins and phospholipids present in the pulp. However, coconut milk remains physically unstable and subject to phase separation. Hence the need to use stabilizers. The objective of this study was to determine the optimal conditions for stabilizing coconut milk using cashew gum as a stabilizing agent, by the methodology of response surfaces using a 32 full factorial design. Thus, the effects of the concentration of cashew tree gum and pulp/water ratio on some physicochemical parameters of coconut milk such as creaming index,
\end{abstract}


droplet size index, viscosity and turbidity loss rate were evaluated. The results indicated that the optimum conditions were $1.50 \%$ for the cashew tree gum concentration and $1 / 1(\mathrm{~g} / \mathrm{mL})$ for the pulp/water ratio. The optimal responses recorded were $60.239 \%$; $0.716 ; 0.076 \mathrm{mPa} . \mathrm{s}$ and $0.824 \AA$ 解 for creaming index, droplet size index, viscosity and turbidity loss rate respectively. Furthermore, no significant difference ( $p>0.05$ ) was observed between the experimental values and the predicted values. The different models developed to predict the different coconut milk characteristics were very significant, with high determination coefficients ( $>0.95$ ) and insignificant lacks of fit ( $p>0.05$ ). Cashew gum would therefore have a strong potential in stabilizing emulsions.

Keywords: Coconut Milk, Stabilization, Cashew Tree Gum, Optimization, Response Surface Methodology

\section{Introduction}

De nombreux produits culinaires et de confiserie sont caractérisés par une structure polyphasée. Les phases peuvent différer par leur contenu, leur état global, leurs ingrédients et leur degré de dispersion. Ces systèmes alimentaires sont thermodynamiquement instables et nécessitent le développement de solutions efficaces pour renforcer la stabilité de leurs processus (Goralchuk et al., 2019).

Le lait de coco est l'un des exemples d'émulsion du type huile-danseau $(\mathrm{H} / \mathrm{E})$. Son apparence a été décrite comme le liquide blanc opaque obtenu par pressage à partir de l'endosperme de noix de coco (Cocos nucifera) râpée ; avec ou sans addition d'eau (Tangsuphoom \& Coupland, 2005). Il contient environ 54\% d'humidité, $35 \%$ de matières grasses et $11 \%$ de matières solides non grasses (Simuang et al., 2004). Habituellement, la séparation d'une émulsion en phases crémeuse et aqueuse se produit après un certain temps de repos, ce qui conduit au défaut physique du lait de coco (Jirapeangtong et al., 2008). Tangsuphoom et Coupland (2008) ont déclaré que l'émulsion de lait de coco était relativement instable en raison de la grande taille des gouttelettes. Une autre étude a rapporté que les mauvaises propriétés émulsifiantes des protéines du lait de coco adsorbées aux interfaces huile-eau (H-E) contribueraient également à l'instabilité du lait de coco (Onsaard et al., 2006). Chauffer le lait de coco à une température plus élevée entraînera une plus grande dénaturation des protéines. Ainsi, des agrégats plus importants de gouttelettes d'huile ont été observés (Peamprasart \& Chiewchan, 2006). Généralement, la dénaturation des protéines se produit lors du chauffage du lait de coco à $80{ }^{\circ} \mathrm{C}$. Tandis que le chauffage du lait de coco à $90-95{ }^{\circ} \mathrm{C}$ pendant plusieurs minutes pourrait détruire la plupart des protéines (Onsaard et al., 2006 ; Peamprasart \& Chiewchan, 2006 ; Tangsuphoom \& Coupland, 2008). Par conséquent, pour une conservation à long terme du lait de coco, il 
est nécessaire de le stabiliser par l'addition d'émulsifiants et de stabilisants appropriés suivi d'une homogénéisation pour réduire la taille des gouttelettes lipidiques.

La stabilité physique des émulsions est influencée par plusieurs facteurs, tels que la teneur en matière grasse, la taille des gouttelettes lipidiques, le type d'émulsifiant ou stabilisant, le rapport émulsifiant/matière grasse (Simuang et al., 2004 ; Tangsuphoom \& Coupland, 2005 ; Onsaard et al., 2006 ; Peamprasart \& Chiewchan, 2006 ; Jirapeangtong et al., 2008 ; Tangsuphoom \& Coupland, 2008). Différents types d'émulsifiants, tels que des tensioactifs (monoglycérides et leurs esters, phospholipides), des protéines (caséinate de sodium) et particulièrement des polysaccharides (gommes) sont utilisés pour stabiliser les émulsions (Noorlaila et al., 2015 ; Jiang et al., 2016 ; Fatimah et al., 2017 ; Abdullah et al., 2018). Cependant, aucune étude n'a été rapporté à notre connaissance, sur l'utilisation de la gomme de cajou (Anacardium occidentale) comme stabilisant du lait de coco.

Ainsi, la présente étude a été entreprise dans le but d'étudier l'influence de la concentration en gomme de cajou et du ratio pulpes/eau sur certains paramètres de stabilité des laits de coco tels que l'indice de crémage, l'indice de taille des gouttelettes, la viscosité et le taux de perte de turbidité. Les conditions optimales de stabilité ont également été déterminées.

\section{Matériel et Méthodes Matériel}

Les noix de coco (Cocos nucifera L.) matures, de la variété Grand Ouest Africain (GOA), ont été achetées dans un marché local de la commune de Yopougon (Abidjan, Côte d'Ivoire). Quant à la Gomme de Cajou (GC), extraite des exsudats d'anacardier (Anacardium occidentale L.), elle a été obtenue auprès du Laboratoire de Biocatalyse et Bioprocédés (LBB) de l'Université NANGUI ABROGOUA (Abidjan, Côte d'Ivoire).

\section{Production du lait de coco stabilisé}

La production du lait de coco stabilisé a été effectuée selon la méthode décrite par Ekanem et Ojimelukwe (2017) et Noorlaila et al. (2015) respectivement. Ainsi, des pulpes fraîches de coco ont été obtenues après débourrage, fendage et extraction à partir des noix de cocos matures. Ces pulpes ont ensuite été découpées en petits morceaux, rincées à l'eau, puis blanchies à $80^{\circ} \mathrm{C}$ pendant $1 \mathrm{~min}$. Cette pulpe blanchie a ensuite été broyée dans un mixeur avec ajout d'eau préalablement chauffée à $60^{\circ} \mathrm{C}$ selon un rapport pulpe/eau $(\mathrm{m} / \mathrm{v})$ de $1 / 3,1 / 2$ et $1 / 1$. Le mélange ainsi obtenu, a été filtré à travers un filtre à café $\mathrm{N}^{\circ} 4(\mathrm{KONOS} \mathrm{GmbH})$, puis le filtrat obtenu, a été pasteurisé à $80^{\circ} \mathrm{C}$ pendant $4 \mathrm{~min}$. A ce lait pasteurisé, du mucilage de gomme de cajou a été ajouté jusqu'à ce que les rapports pulpe/eau soient respectés et les laits de 
coco stabilisés aient des concentrations $(\mathrm{m} / \mathrm{v})$ respectives de $1 ; 1,25$ et $1,50 \%$ en gomme de cajou. Le mélange ainsi obtenu, a été soumis à une agitation lente pendant $5 \mathrm{~min}$, puis homogénéisé à $4200 \mathrm{tr} / \mathrm{min}$ durant $3 \mathrm{~min}$ pour donner un lait de coco stabilisé comme illustré à la Figure 1.

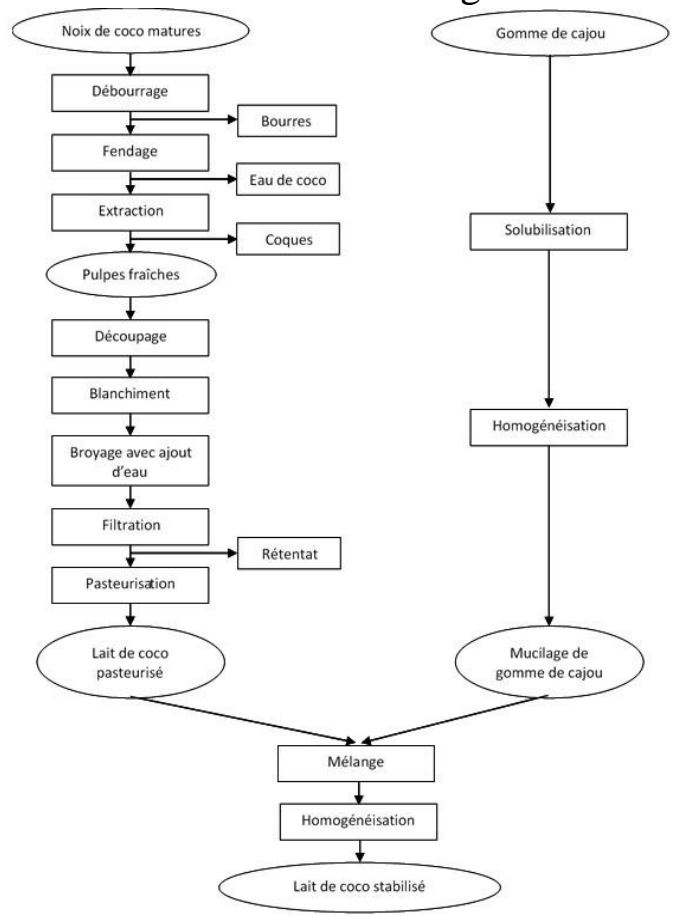

Figure 1. Diagramme de production du lait de coco stabilisé

\section{Techniques d'analyses}

\section{Indice de crémage}

L'indice de crémage (IC) du lait de coco stabilisé a été déterminé selon la méthode décrite par Owens et al. (2018). Trente (30) mL de lait ont été transféré dans un tube de $50 \mathrm{~mL}$ puis fermé pour éviter l'évaporation et laisser à température ambiante. Les mesures de stabilité ont été effectuées chaque 48 $\mathrm{h}$ pendant 14 jours en raison de 6 mesures de taille par séance. L'indice de crémage (IC) a été calculé selon la formule mathématique suivante :

$$
\mathrm{IC}(\%)=\frac{\mathrm{T}_{\mathrm{S}}}{\mathrm{T}_{\mathrm{E}}} \times 100
$$

TE : taille totale de l'émulsion et TS : taille du sérum

\section{Indice de taille des gouttelettes (ITG)}

La mesure de l'indice de taille des gouttelettes est déterminée par la méthode décrite par Mirhosseini et al. (2008). L'échantillon est dilué avec de l'eau distillée au ratio $(1 / 100)$ et homogénéisé. L'absorbance a été déterminé immédiatement à 400 et $800 \mathrm{~nm}$ à l'aide un spectrophotomètre UV-visible 
l'eau distillée est choisi comme témoin. La valeur du rapport (Équation 2) de l'absorbance à $\lambda=800 \mathrm{~nm}$ et $\lambda=400 \mathrm{~nm}$ permet de déterminer l'indice de taille des gouttelettes (ITG).

$$
\text { ITG }=\frac{\operatorname{Absorbance}\left(\lambda_{800}\right)}{\operatorname{Absorbance}\left(\lambda_{400}\right)}
$$

\section{Viscosité}

La mesure de la viscosité du lait de coco a été effectuée selon la méthode décrite par Arioui et al. (2018) avec un viscosimètre Brookfield à chute à bille de type KF40 équipé d'un thermostat. Quarante (40) $\mathrm{mL}$ de lait de coco prélevé a été transféré dans le tube et maintenue à température constante à $39^{\circ} \mathrm{C}$. On mesure le temps nécessaire à la chute d'une bille soumise à la gravité dans lait de coco. La viscosité est déterminée selon l'expression mathématique suivante :

$$
\eta(\mathrm{mPa} . \mathrm{s})=\frac{2 \mathrm{r}^{2} \mathrm{~g}\left(\rho-\rho^{\prime}\right) \mathrm{t}}{91}
$$

Avec $\boldsymbol{\eta}=$ Coefficient de viscosité dynamique de l'émulsion, $\mathrm{r}=$ Rayon de la bille, $\boldsymbol{\rho}=$ Masse volumique de la bille, $\boldsymbol{\rho}^{\prime}=$ Masse volumique du lait, $1=$ Distance de chute et $\mathrm{t}=$ Temps de chute.

\section{Taux de perte de turbidité}

Le taux de perte de turbidité des laits de coco stabilisé en condition diluée a été déterminé selon la méthode décrite par Chang et al. (2018). Les laits ont d'abord été dilués à $0,25 \%(\mathrm{v} / \mathrm{v})$ dans de l'eau distillée et stockés dans des bouteilles en plastique de $1,5 \mathrm{~L}$ à température ambiante avant la lecture des absorbances à la longueur d'onde $\lambda=500 \mathrm{~nm}$ à l'aide d'un spectrophotomètre UV-visible (UV-2101PC, Shimadzu Corp., Tokyo, Japon). Des mesures quotidiennes ont été effectuées jusqu'à ce qu'une réduction de $10 \%$ de la valeur initiale contre de l'eau distillée soit obtenue. Un modèle de premier ordre a été appliqué en ajustant les données (absorbance en fonction du temps) pour chaque échantillon afin de dériver le taux de perte de turbidité (k1, Å/jr) selon la relation mathématique suivante :

$$
\ln \mathrm{A}_{\mathrm{t}}=\ln \mathrm{A}_{0}-\mathrm{k}_{1} \mathrm{t}
$$

Avec, $\mathrm{t}=$ temps $; \mathrm{A}_{\mathrm{t}}=$ absorbance au temps $\mathrm{t} ; \mathrm{A}_{0}=$ absorbance au temps $\mathrm{t}=0$ et $\mathrm{k}_{1}=$ constante de vitesse du premier ordre.

\section{Planification expérimentale}

La Méthodologie des Surfaces de Réponses (MSR) a été appliquée dans la présente expérience pour évaluer les effets isolés et les interactions possibles entre les différents facteurs identifiés sur certaines caractéristiques physiques (réponses observées) des laits de coco stabilisés. Les effets de deux 
variables indépendantes, à savoir la concentration en gomme de cajou (1$1,5 \%, \mathrm{~m} / \mathrm{v})$ et le ratio pulpe-eau $(1 / 3-1 / 1, \mathrm{~m} / \mathrm{v})$ ont été étudiés en relation avec les caractéristiques physiques, telles que l'indice de crémage, l'indice de taille des gouttelettes, la viscosité et le taux de perte de turbidité. Les variables indépendantes et leurs différentes modalités, qui ont été choisies étaient choisis en se basant sur les études se référant à la formation, la stabilisation des émulsions du types $\mathrm{H} / \mathrm{E}$ et sur celle de l'extraction de lait de coco (McClements, 2015 ; Fatimah, et al., 2017) sont présentés dans le tableau 1.

Tableau 1. Variables indépendantes et leurs niveaux (Réelles et codées)

\begin{tabular}{|l|l|l|l|l|l|}
\hline Variables indépendantes & & Niveaux \\
\hline Réelles & Codées & & $-\mathbf{1}$ & $\mathbf{0}$ & $\mathbf{+ 1}$ \\
\hline $\begin{array}{l}\text { Concentrations en gomme de } \\
\text { cajou }(\mathrm{g} / \mathrm{mL})\end{array}$ & $\mathrm{X}_{1}$ & & 1 & 1,25 & 1,50 \\
\hline Ratios pulpes/eau $(\mathrm{g} / \mathrm{mL})$ & $\mathrm{X}_{2}$ & & $1 / 3$ & $1 / 2$ & $1 / 1$ \\
\hline
\end{tabular}

Le plan factoriel complet $3 \mathrm{k}$ ( $\mathrm{k}$ est le nombre de facteurs de l'étude et 3 indique le nombre de niveaux par facteur) a été utilisé dans le but de déterminer les conditions optimales pour la stabilisation du lait de coco avec la gomme de cajou. Le nombre d'essais nécessaire pour cette étude est $\mathrm{N}=3 \mathrm{k}$. Etant donné que nous avons 2 facteurs, le plan d'expérience sera une matrice de $32=9$ essais et une répétition des points centraux ce qui donne au total 10 essais (Montgomery, 2017). Afin d'étudier la relation entre les variables indépendantes et chacune des réponses étudiées et également, de prédire les points optimaux, une fonction polynomiale du second ordre a été élaborée pour chaque réponse. La forme générale de l'équation polynomiale du second ordre est donnée par la formule mathématique suivante :

$$
\mathrm{Y}=\beta_{0}+\sum_{\mathrm{i}=1}^{\mathrm{j}} \beta_{\mathrm{i}} \mathrm{X}_{\mathrm{i}}+\sum_{\mathrm{i}=1}^{\mathrm{jf} \mathrm{i}} \beta_{\mathrm{ij}} \mathrm{X}_{\mathrm{i}} \mathrm{X}_{\mathrm{j}}+\sum_{\mathrm{i}=1}^{\mathrm{j}} \beta_{\mathrm{ii}} \mathrm{X}_{\mathrm{i}}^{2}
$$

Avec $\mathrm{Y}$ - réponses prédites ; $\beta 0$ - termes d'interceptions ; $\beta \mathrm{i}$ - coefficients linéaires ; $\beta \mathrm{ij}$ coefficients d'interaction; $\beta \mathrm{ii}$ - coefficient quadratique, et Xi $(\mathrm{i}=1)$ et $\mathrm{Xj}(\mathrm{j}=2)$ - variables indépendantes codés.

\section{Analyses statistiques}

Les matrices d'expériences, les différentes procédures d'optimisation, les différents graphiques, ainsi que l'analyse de l'ensemble des données collectées lors de cette étude ont été élaborés à l'aide du logiciel STATISTICA (Version 10 Statsoft Inc, Tulsa-USA Headquaters). La détermination des coefficients $(\beta)$ des différents modèles de cette étude a été réalisée par une approche matricielle en utilisant une Régression Linéaire Multiple (Montgomery, 2017). L'évaluation de la qualité globale des modèles développés a été réalisée à l'aide d'une part, d'une ANOVA utilisant le test de Fisher-Snedecor et la valeur de la probabilité associée (significativité du modèle) ; et d'autre part, du calcul des coefficients de détermination $\left(\mathrm{R}^{2}\right)$ et de 
détermination ajusté (adj- $\mathrm{R}^{2}$ ) qui mesurent la qualité de l'ajustement du modèle de régression. La significativité des effets linéaires, quadratiques et des interactions des différents facteurs; ainsi que celle de chacun de ces coefficients a été évaluée par la comparaison entre la probabilité observée (pvaleur) et une probabilité critique $(\mathrm{P}=0,05)$. Ainsi, lorsque $\mathrm{p}$-valeur $\leq 0,05$, on conclut que l'effet est significatif (Baş \& Boyac1, 2007). L'optimisation de la ou des réponse(s) a été faite selon l'approche de la fonction de désirabilité décrite par Derringer et Suich (1980).

\section{Résultats et Discussion}

Les résultats de l'effet de la concentration en gomme de cajou $\left(\mathrm{X}_{1}\right)$ et du ratio pulpes/eau $\left(\mathrm{X}_{2}\right)$ sur l'indice de crémage $\left(\mathrm{Y}_{1} ; \%\right)$, l'indice de taille des gouttelettes $\left(\mathrm{Y}_{2}\right)$, la viscosité $\left(\mathrm{Y}_{3} ; \mathrm{mPa} . \mathrm{s}\right)$ et le taux de perte de turbidité $\left(\mathrm{Y}_{4}\right.$;

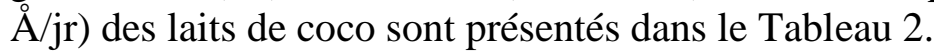

Ces résultats montrent que les indices de crémages $\left(\mathrm{Y}_{1} ; \%\right)$ sont compris entre 60,30 et $83 \%$; avec le plus petit indice $(60,30 \%$ ) obtenu pour une concentration en gomme de cajou $\left(\mathrm{X}_{1}\right)$ de $1,50 \%$ et un ratio pulpes/eau $\left(\mathrm{X}_{2}\right)$ de $1 / 1$. En ce qui concerne l'indice de taille des gouttelettes $\left(\mathrm{Y}_{2}\right)$, les valeurs sont comprises entre 0,513 et 0,903 , avec le plus faible indice $(0,513)$ obtenu pour une concentration en gomme de cajou $\left(\mathrm{X}_{1}\right)$ de $1,50 \%$ et un ratio pulpes/eau $\left(\mathrm{X}_{2}\right)$ de $1 / 2$. La viscosité $\left(\mathrm{Y}_{3} ; \mathrm{mPa} . \mathrm{s}\right)$ des différents laits de coco obtenus, varie entre 0,0351 et 0,0761 (mPa.s). La viscosité la plus élevée $(0,0761 \mathrm{mPa} . \mathrm{s})$ a été obtenue pour une concentration en gomme de cajou $\left(\mathrm{X}_{1}\right)$ de $1,50 \%$ et un ratio pulpes/eau $\left(\mathrm{X}_{2}\right)$ de $1 / 1$. Enfin, les taux de perte de turbidité ( $\left.\mathrm{Y}_{4} ; \AA / \mathrm{\AA} / \mathrm{jr}\right)$ sont compris entre 0,795 et $1,189(\AA / \mathrm{d} / \mathrm{jr})$. Le plus petit taux de perte de turbidité $(0,795 \AA$ Åjr) a été obtenu pour une concentration en gomme de cajou $\left(\mathrm{X}_{1}\right)$ de $1 \%$ et un ratio pulpes/eau $\left(\mathrm{X}_{2}\right)$ de $1 / 1$. La MSR a été largement appliqué dans des expériences similaires dans le domaine des sciences alimentaires lorsque plusieurs facteurs sont à l'étude, et elle a été signalé comme étant particulièrement utile (Mirhosseini, et al., 2008; Gharibzahedi, et al., 2012 ; Noorlaila et al., 2015).

Tableau 2. Matrice d'expérimentation et données expérimentales obtenues

\begin{tabular}{|l|l|l|l|l|l|l|}
\hline \multirow{2}{*}{ Essais } & \multicolumn{4}{l|}{$\begin{array}{l}\text { Variables } \\
\text { indépendantes }\end{array}$} & \multicolumn{4}{l|}{ Variables dépendantes } \\
\cline { 2 - 7 } & $\mathbf{X}_{\mathbf{1}}$ & $\mathbf{X}_{\mathbf{2}}$ & $\mathbf{Y}_{\mathbf{1}}(\boldsymbol{\%})$ & $\mathbf{Y}_{\mathbf{2}}$ & $\mathbf{Y}_{\mathbf{3}}(\mathbf{m P a} . \mathbf{s})$ & $\mathbf{Y}_{\mathbf{4}}(\mathbf{A} / \mathbf{j r})$ \\
\hline $\mathbf{1}$ & $-1(1)$ & $-1(1 / 3)$ & $83,00 \pm 0,16$ & $0,831 \pm 0,003$ & $0,0351 \pm 0,0003$ & $1,139 \pm 0,006$ \\
\hline $\mathbf{2}$ & $\begin{array}{l}+1 \\
(1,5)\end{array}$ & $-1(1 / 3)$ & $80,14 \pm 0,06$ & $0,701 \pm 0,005$ & $0,0502 \pm 0,0008$ & $1,189 \pm 0,002$ \\
\hline $\mathbf{3}$ & $-1(1)$ & $\begin{array}{l}+1 \\
(1 / 1)\end{array}$ & $63,10 \pm 0,10$ & $0,903 \pm 0,009$ & $0,0581 \pm 0,0004$ & $0,795 \pm 0,012$ \\
\hline $\mathbf{4}$ & $+1(1,5)$ & $\begin{array}{l}+1 \\
(1 / 1)\end{array}$ & $60,30 \pm 0,09$ & $0,704 \pm 0,008$ & $0,0761 \pm 0,0006$ & $0,821 \pm 0,016$ \\
\hline
\end{tabular}




\begin{tabular}{|l|l|l|l|l|l|l|}
\hline $\mathbf{5}$ & $-1(1)$ & $0(1 / 2)$ & $68,15 \pm 0,19$ & $0,698 \pm 0,006$ & $0,0367 \pm 0,0004$ & $1,101 \pm 0,008$ \\
\hline $\mathbf{6}$ & $\begin{array}{l}+1 \\
(1,5)\end{array}$ & $0(1 / 2)$ & $65,07 \pm 0,04$ & $0,513 \pm 0,004$ & $0,0599 \pm 0,0005$ & $1,119 \pm 0,005$ \\
\hline $\mathbf{7}$ & $0(1,25)$ & $-1(1 / 3)$ & $81,90 \pm 0,21$ & $0,784 \pm 0,001$ & $0,0415 \pm 0,0003$ & $1,171 \pm 0,014$ \\
\hline $\mathbf{8}$ & $0(1,25)$ & $\begin{array}{l}+1 \\
(1 / 1)\end{array}$ & $61,78 \pm 0,17$ & $0,810 \pm 0,005$ & $0,0619 \pm 0,0009$ & $0,811 \pm 0,01$ \\
\hline $\mathbf{9}$ & $0(1,25)$ & $0(1 / 2)$ & $66,47 \pm 0,26$ & $0,611 \pm 0,004$ & $0,0471 \pm 0,0007$ & $1,105 \pm 0,03$ \\
\hline $\mathbf{1 0}$ & $0(1,25)$ & $0(1 / 2)$ & $66,64 \pm 0,34$ & $0,625 \pm 0,002$ & $0,0464 \pm 0,0004$ & $1,112 \pm 0,007$ \\
\hline
\end{tabular}

$X_{I}=$ concentration de la gomme de cajou $(\mathrm{g} / \mathrm{mL}) ; X_{2}=$ ratio de préparation du lait de coco $(\mathrm{g} / \mathrm{mL}) ; Y_{1}=$ indice de crémage $(\%) ; Y_{2}=$ indice de taille des gouttelettes $; Y_{3}=$ viscosité (mPa.s) ; $Y_{4}=$ taux de perte de turbidité $(\AA / j r)$ Ces valeurs sont exprimées en tant que Moyenne \pm Ecart type ( $n=3$ détermination).

\section{Influence des variables indépendantes sur les caractéristiques du lait de coco}

Les effets des deux variables indépendantes [la concentration en gomme de cajou $\left(\mathrm{X}_{1}\right)$ et le ratio pulpes/eau $\left(\mathrm{X}_{2}\right)$ ] sur les caractéristiques physiques des laits de coco sont illustrés par les courbes d'isoréponses (Figure 2) et les diagrammes de Pareto (Figure 3). Pour l'indice de crémage, les courbes d'isoréponses ont montré que ce paramètre diminue au fur et à mesure que les variables indépendantes augmentent dans leur domaine expérimental. Le plus petit indice de crémage a été obtenu pour des concentrations en gomme comprise entre 1,20 et 1,50\% et des ratios pulpes/eau de 1/2 à $1 / 1$ (Figure 2A). Par ailleurs, le diagramme de Pareto a montré une influence statistiquement significative $(\mathrm{p} \leq 0,05)$ de ces deux variables indépendantes sur l'indice de crémage (Figure 3A). Ces résultats sont en accord avec ceux rapportés par Owens et al. (2018). Les courbes d'isoréponses ont montré une diminution de l'indice de taille des gouttelettes avec l'augmentation de la concentration en gomme de cajou $\left(\mathrm{X}_{1}\right)$ et une diminution du ratio pulpe/eau $\left(\mathrm{X}_{2}\right)$. Les valeurs minimales ont été obtenues pour des concentrations en gomme de cajou comprises entre 1,20 et $1,50 \%$ et des ratios pulpes/eau qui varient de $1 / 3$ à $1 / 2$ (Figure 2B). Un effet statistiquement significatif $(p \leq 0,05)$ de la concentration en gomme de cajou sur l'indice de taille des gouttelettes a été observé (Figure 3B). Des effets similaires ont été observés par Gharibzahedi et al. (2012), qui ont évalué l'influence de la concentration en gomme arabique et de la teneur d'une huile extraite des noix (Juglans regia). En ce qui concerne la viscosité, nos résultats (Figure $2 \mathrm{C}$ ) ont montré une augmentation de ce paramètre pour toute augmentation de la concentration en gomme de cajou $\left(\mathrm{X}_{1}\right)$ et du ratio pulpes/eau $\left(\mathrm{X}_{2}\right)$. De plus, le diagramme de Pareto (Figure 3C) a montré des effets statistiquement significatifs $(\mathrm{p} \leq 0,05)$ des deux variables sur la viscosité (Mirhosseini et al., 2008). Les courbes d'isoréponses (Figure 2D), illustrant l'évolution de taux de perte de turbidité $\left(\mathrm{Y}_{4} ; \AA / j r\right)$ en fonction de la 
concentration en gomme de cajou $\left(\mathrm{X}_{1}\right)$ et du ratio pulpes/eau $\left(\mathrm{X}_{2}\right)$, ont montré que le taux de perte de turbidité augmente avec l'augmentation de la concentration en gomme de cajou $\left(\mathrm{X}_{1}\right)$ et du ratio pulpes/eau $\left(\mathrm{X}_{2}\right)$. Une influence statistiquement significative $(\mathrm{p} \leq 0,05)$ de ces deux facteurs sur le taux de perte de turbidité a par ailleurs été observée sur le diagramme de Pareto (Figure 3D). Cette observation a été rapportée par Mirhosseini et al. (2008) et Gharibzahedi et al. (2012).
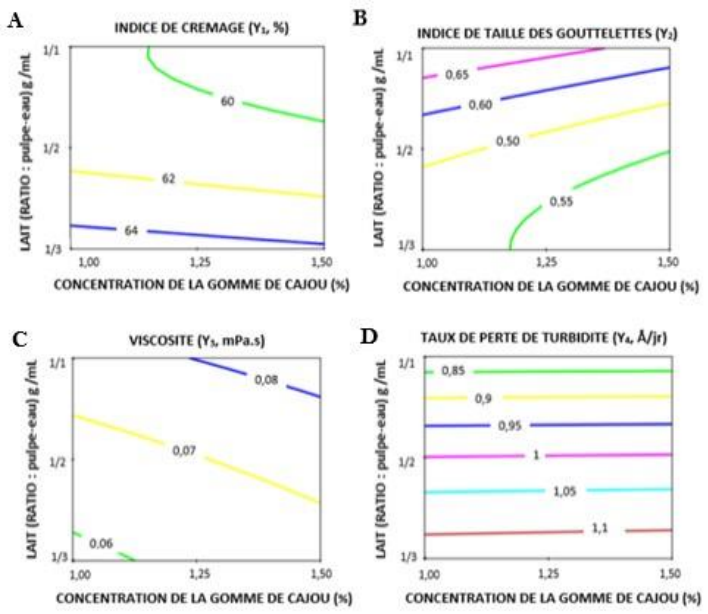

Figure 2. Courbes d'isoréponses illustrant respectivement les effets combinés des facteurs $\mathrm{X}_{1}$ et $\mathrm{X}_{2}$ sur l'indice de taille de crémage $\left(\mathrm{Y}_{1}\right)$, l'indice de taille des gouttelettes $\left(\mathrm{Y}_{2}\right)$, la viscosité $\left(\mathrm{Y}_{3}\right)$ et le taux de perte de turbidité $\left(\mathrm{Y}_{4}\right)$.
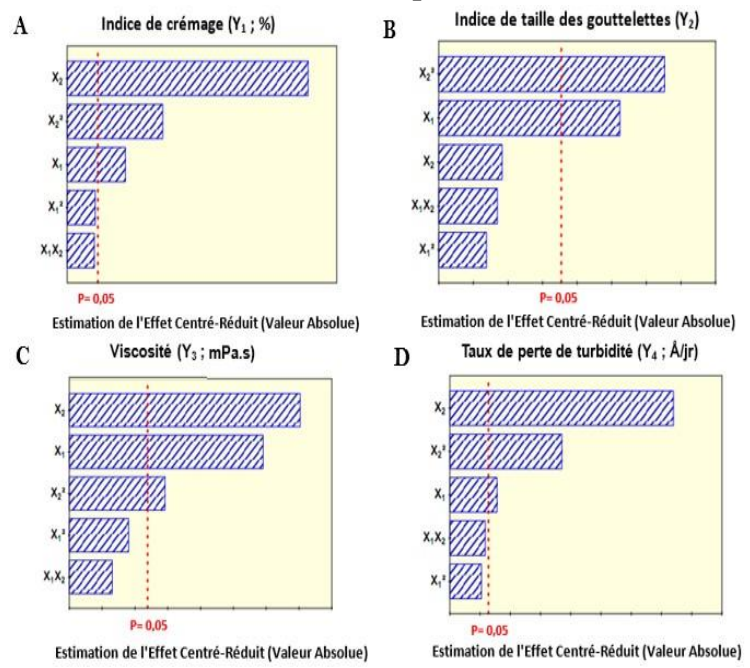

Figure 3. Diagrammes de Pareto illustrant respectivement les effets combinés des facteurs $\mathrm{X}_{1}$ et $\mathrm{X}_{2}$ sur l'indice de crémage $\left(\mathrm{Y}_{1}\right)$, sur l'indice de taille des gouttelettes $\left(\mathrm{Y}_{2}\right)$, sur la viscosité $\left(\mathrm{Y}_{3}\right)$ et le taux de perte de turbidité $\left(\mathrm{Y}_{4}\right)$. 


\section{Construction et évaluation de la qualité d'ajustement des modèles}

Les résultats de l'estimation et de la significativité des coefficients de régression des modèles du second ordre, représentant la relation entre les différentes variables dépendantes [l'indice de crémage $\left(\mathrm{Y}_{1} ; \%\right)$, l'indice de taille des gouttelettes $\left(\mathrm{Y}_{2}\right)$, la viscosité $\left(\mathrm{Y}_{3} ; \mathrm{mPa} . \mathrm{s}\right)$ et le taux de perte de turbidité $\left.\left(\mathrm{Y}_{4} ; \AA ̊ \AA / \mathrm{j} \mathrm{r}\right)\right]$ et les deux facteurs étudiés [la concentration en gomme de cajou $\left(\mathrm{X}_{1}\right)$ et le ratio pulpes/eau $\left(\mathrm{X}_{2}\right)$ ] sont présentés dans le Tableau 3.

Ces résultats ont montré que tous les coefficients de régressions sont significatifs $(p \leq 0,05)$ sauf les coefficients quadratique $\left(\beta_{11}\right)$ et d'interaction $\left(\beta_{12}\right)$ qui sont non significatifs $(\mathrm{p}>0,05)$ pour l'indice de crémage $\left(\mathrm{Y}_{1} ; \%\right)$, la viscosité $\left(\mathrm{Y}_{3} ; \mathrm{mPa} . \mathrm{s}\right)$ et le taux de perte de turbidité $\left(\mathrm{Y}_{4} ; \AA / \mathrm{A} / \mathrm{jr}\right)$. Tandis que pour l'indice de taille des gouttelettes $\left(\mathrm{Y}_{2}\right)$, seuls les coefficients linéaire $\left(\beta_{2}\right)$; quadratique $\left(\beta_{11}\right)$ et d'interaction $\left(\beta_{12}\right)$ sont non significatifs $(p>0,05)$ (Tableau $3)$.

Les modèles polynomiaux raffinés du second ordre, exprimant respectivement l'indice de crémage $\left(\mathrm{Y}_{1} ; \%\right)$, l'indice de taille des gouttelettes $\left(\mathrm{Y}_{2}\right)$, la viscosité $\left(\mathrm{Y}_{3} ; \mathrm{mPa} . \mathrm{s}\right)$ et le taux de perte de turbidité $\left(\mathrm{Y}_{4} ; \AA / \mathrm{A} \mathrm{j}\right)$ en fonction des variables indépendantes [la concentration en gomme de cajou $\left(\mathrm{X}_{1}\right)$ et le ratio pulpes/eau $\left.\left(\mathrm{X}_{2}\right)\right]$ sont donnés par les relations mathématiques suivantes :

$$
\begin{gathered}
\mathrm{Y}_{1}(\%)=66,63+1,46 \mathrm{X}_{1}-9,98 \mathrm{X}_{2}+5,14 \mathrm{X}_{2}^{2} \\
\mathrm{Y}_{2}=0,62-0,08 \mathrm{X}_{1}+0,18 \mathrm{X}_{2}^{2} \\
\mathrm{Y}_{3}(\mathrm{mPa} . \mathrm{s})=0,046+0,009 \mathrm{X}_{1}+0,011 \mathrm{X}_{2}+0,006 \mathrm{X}_{2}^{2} \\
\mathrm{Y}_{4}(\stackrel{0}{\mathrm{~A}} / \mathrm{jr})=1,11+0,016 \mathrm{X}_{1}-0,179 \mathrm{X}_{2}-0,121 \mathrm{X}_{2}^{2}
\end{gathered}
$$

L'analyse de variance (ANOVA) sur la qualité d'ajustement des modèles polynomiaux raffinés du second ordre a montré que ces modèles sont tous significatifs $(p \leq 0,05)$ avec des manques d'ajustement non-significatifs (p>0,05) (Tableau 4). Par ailleurs l'analyse graphique (Figure 4), représentant les variables dépendantes théoriques en fonction des variables dépendantes mesurées, a montré une bonne corrélation entre les réponses théoriques et mesurées, avec des coefficients $\left(\mathrm{R}^{2}=0,9849-0,9998, \mathrm{R}^{2}\right.$ (ajusté) $=0,9662-$ 0,9996) supérieurs à 0,80 (Baş \& Boyac1, 2007 ; Montgomery, 2017).

Tableau 3. Estimation des coefficients de régression linéaires, quadratiques et d'interaction des modèles prédictifs pour les variables dépendantes

\begin{tabular}{|l|l|l|l|l|l|}
\hline $\begin{array}{l}\text { Variables } \\
\text { dépendantes }\end{array}$ & Termes & $\begin{array}{l}\text { Coefficients } \\
\text { estimés }\end{array}$ & ES & t-exp & p-valeur \\
\hline \multirow{4}{*}{$Y_{1}$} & $\boldsymbol{\beta}_{\mathbf{0}}$ & 66,62929 & 0,091975 & 724,428 & $0,000000(*)$ \\
\cline { 2 - 6 } & $\boldsymbol{\beta}_{1}$ & 1,45667 & 0,062831 & $-23,184$ & $0,000021(*)$ \\
\cline { 2 - 6 } & $\boldsymbol{\beta}_{\mathbf{2}}$ & $-9,97667$ & 0,062831 & $-158,786$ & $0,000000(*)$ \\
\cline { 2 - 6 } & $\boldsymbol{\beta}_{11}$ & $-0,09357$ & 0,100754 & $-0,929$ & $0,405605(\mathbf{n s})$ \\
\hline
\end{tabular}




\begin{tabular}{|c|c|c|c|c|c|}
\hline & $\beta_{22}$ & 5,13643 & 0,100754 & 50,980 & $0,000001(*)$ \\
\hline & $\beta_{12}$ & $-0,01500$ & 0,076952 & 0,195 & 0,854950 (ns) \\
\hline \multirow[t]{6}{*}{$\mathbf{Y}_{2}$} & $\beta_{0}$ & 0,617929 & 0,005916 & 104,4490 & $0,006095(*)$ \\
\hline & $\beta_{1}$ & $-0,085667$ & 0,004041 & $-21,1970$ & $0,030011(*)$ \\
\hline & $\beta_{2}$ & 0,016833 & 0,004041 & 4,1652 & 0,150005 (ns) \\
\hline & $\beta_{11}$ & $-0,012357$ & 0,006481 & $-1,9067$ & 0,307499 (ns) \\
\hline & $\beta_{22}$ & 0,179143 & 0,006481 & 27,6423 & $0,023021(*)$ \\
\hline & $\beta_{12}$ & $-0,017250$ & 0,004950 & $-3,4850$ & 0,177893 (ns) \\
\hline \multirow[t]{6}{*}{$\mathbf{Y}_{3}$} & $\beta_{0}$ & 0,046286 & 0,001400 & 33,05350 & $0,000005(*)$ \\
\hline & $\beta_{1}$ & 0,009383 & 0,000957 & 9,80899 & $0,000606(*)$ \\
\hline & $\beta_{2}$ & 0,011550 & 0,000957 & 12,07394 & $0,000270(*)$ \\
\hline & $\beta_{11}$ & 0,002479 & 0,001534 & 1,61578 & 0,181447 (ns) \\
\hline & $\beta_{22}$ & 0,005879 & 0,001534 & 3,83223 & $0,018583(*)$ \\
\hline & $\beta_{12}$ & 0,000725 & 0,001172 & 0,61881 & 0,569540 (ns) \\
\hline \multirow[t]{6}{*}{$\mathbf{Y}_{4}$} & $\beta_{0}$ & 1,110357 & 0,004090 & 271,5137 & $0,000000(*)$ \\
\hline & $\beta_{1}$ & 0,015667 & 0,002794 & 5,6079 & $0,004967(*)$ \\
\hline & $\beta_{2}$ & $-0,178667$ & 0,002794 & $-63,9542$ & $0,000000(*)$ \\
\hline & $\beta_{11}$ & $-0,002214$ & 0,004480 & $-0,4943$ & 0,647023 (ns) \\
\hline & $\beta_{22}$ & $-0,121214$ & 0,004480 & $-27,0578$ & $0,000011(*)$ \\
\hline & $\beta_{12}$ & $-0,006000$ & 0,003422 & $-1,7536$ & 0,154365 (ns) \\
\hline
\end{tabular}

(ES) - erreur standard, t-exp - valeur de student calculée, $\mathrm{p}$-valeur- probabilité associé à la valeur de $t, \beta_{0}$-constante, $\left(\beta_{1}, \beta_{2}\right)$ - coefficients linéaires, $\left(\beta_{11}, \beta_{22}\right)$ - coefficients quadratiques,

$\beta_{12}$ - coefficient d'interaction, (ns) - non significative ( $\left.p>0,05\right),(*)-\operatorname{significatif}(p \leq 0,05)$

Tableau 4. Table d'ANOVA de la qualité d'ajustement des modèles prédictifs pour les variables dépendantes

\begin{tabular}{|c|c|c|c|c|c|}
\hline Source & ddl & SCE & MCE & Fobs $_{\text {obs }}$ & p-value \\
\hline \multicolumn{6}{|c|}{ Indice de crémage $\left(Y_{l}\right)$} \\
\hline Modèle & 5 & 672,8909 & 671,516 & 28334,008 & $0,000000(*)$ \\
\hline Residues & 4 & 0,0947 & 0,0237 & & \\
\hline Manque d'ajustement & 3 & 0,0803 & 0,0268 & 1,861 & $\begin{array}{l}0,484294 \\
(\mathrm{~ns})\end{array}$ \\
\hline Erreur expérimentale & 1 & 0,0144 & 0,0144 & & \\
\hline Total SCE & 9 & 672,9856 & & & \\
\hline \multicolumn{3}{|c|}{$R^{2}=0,9998$} & \multicolumn{3}{|c|}{$R^{2}$ (ajusté) = 0,9996 } \\
\hline \multicolumn{6}{|c|}{ Indice de taille des gouttelettes $\left(Y_{2}\right)$} \\
\hline Modèle & 5 & 0,12254 & 0,122161 & 1720,577 & $0,00609(*)$ \\
\hline Residues & 4 & 0,000282 & 0,000071 & & \\
\hline Manque d'ajustement & 3 & 0,000184 & 0,000061 & 0,62244 & 0,70428 (ns) \\
\hline Erreur expérimentale & 1 & 0,000098 & 0,000098 & & \\
\hline Total SCE & 9 & 0,122822 & & & \\
\hline \multicolumn{3}{|c|}{$R^{2}=0,9977$} & \multicolumn{3}{|c|}{$R^{2}$ (ajusté) $=0,9948$} \\
\hline \multicolumn{6}{|c|}{ Viscosité $\left(Y_{3}\right)$} \\
\hline Modèle & 5 & 0,00144 & 0,001443 & 288,6 & $0,000005(*)$ \\
\hline Residues & 4 & 0,000022 & 0,000005 & & \\
\hline Manque d'ajustement & 3 & 0,000022 & 0,000007 & 17,5 & $\begin{array}{l}0,134229 \\
\text { (ns) }\end{array}$ \\
\hline Erreur expérimentale & 1 & 0,000000 & 0,000000 & & \\
\hline
\end{tabular}




\begin{tabular}{|l|l|l|l|l|l|}
\hline Total SCE & 9 & 0,001462 & & \\
\hline \multicolumn{7}{|c|}{$\mathbf{R}^{\mathbf{2}}=\mathbf{0 , 9 8 4 9}$} & \multicolumn{3}{c|}{$\mathbf{R}^{\mathbf{2}}$ (ajusté) = 0,9662 } \\
\hline \multicolumn{7}{|c|}{ Taux de perte de turbidité $\left(Y_{4}\right)$} \\
\hline Modèle & 5 & 0,228637 & 0,227345 & 4837,127 & $0,00000\left(^{*}\right)$ \\
\hline Residues & 4 & 0,000187 & 0,000047 & & \\
\hline Manque d'ajustement & 3 & 0,000163 & 0,000054 & 2,16 & $0,45023(\mathrm{~ns})$ \\
\hline Erreur expérimentale & 1 & 0,000025 & 0,000025 & & \\
\hline Total SCE & 9 & 0,228824 & & & \\
\hline \multicolumn{7}{|c|}{$\mathbf{R}^{2}=\mathbf{0 , 9 9 9 1}$} & \multicolumn{5}{c|}{$\mathbf{R}^{\mathbf{2}}$ (ajusté) $=\mathbf{0 , 9 9 8 1}$} \\
\hline
\end{tabular}

ddl - degrés de liberté, SCE - somme des carrés des écarts, MCE - moyennes des carrés des écarts, $\mathrm{F}_{\mathrm{obs}}$ - valeur de Fisher calculée, p-value - probabilité associée à la valeur de $\mathrm{F}, \mathrm{R}^{2}$ coefficient de détermination, $\mathrm{R}^{2}$ (ajusté) - coefficient de détermination ajusté, (ns) - non significative $(\mathrm{p}>0,05)$,

$(*)$ - significatif $(\mathrm{p} \leq 0,05)$.
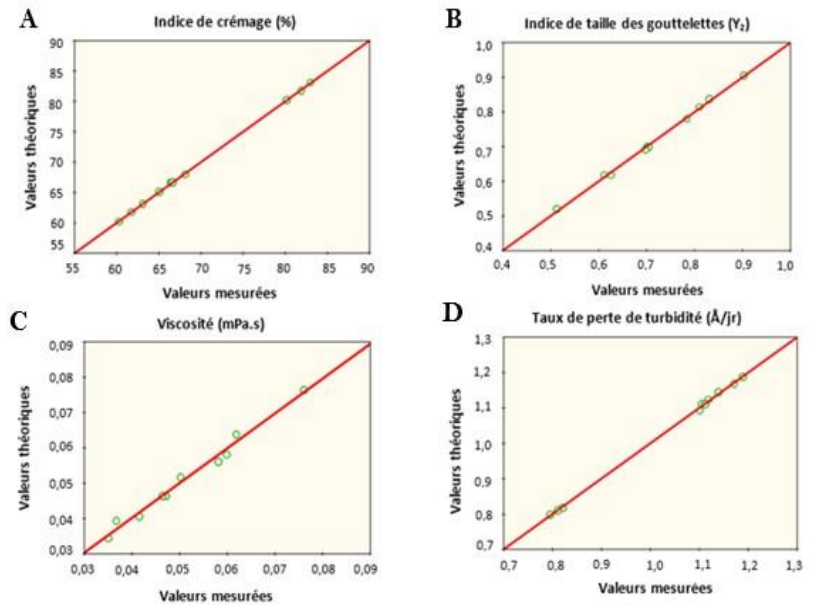

Figure 4. Représentations graphiques des réponses théoriques en fonction des réponses mesurées des laits de coco pour chacune des réponses étudiées

\section{Détermination et vérification des conditions optimales}

Les procédures d'optimisations ont indiqué que les conditions optimales étaient de $1,50 \%$ pour la concentration en gomme de cajou et de $(1 / 1 ; \mathrm{g} / \mathrm{mL})$ pour le ratio pulpes/eau. Les réponses optimales enregistrées ont été de $60,239 \%$; 0,$716 ; 0,076 \mathrm{mPa} . \mathrm{s}$ et de $0,824 \AA / \mathrm{jr}$ respectivement pour l'indice de crémage $\left(\mathrm{Y}_{1}\right)$, l'indice de taille des gouttelettes $\left(\mathrm{Y}_{2}\right)$, la viscosité $\left(\mathrm{Y}_{3}\right)$ et le taux de perte de turbidité $\left(\mathrm{Y}_{4}\right)$ (Figure 5).

Des expériences de confirmation ont en outre été réalisées en triplicata en utilisant les niveaux optimums de $1,50 \%$ pour la concentration en gomme de cajou et de $1 / 1(\mathrm{~g} / \mathrm{mL})$ pour le ratio pulpes/eau. Les résultats expérimentaux ont été de $60,30 \% ; 0,704 ; 0,0761 \mathrm{mPa} . \mathrm{s}$ et de $0,821 \AA / \mathrm{j}$ r respectivement pour l'indice de crémage $\left(\mathrm{Y}_{1}\right)$, l'indice de taille de gouttelettes $\left(\mathrm{Y}_{2}\right)$, la viscosité $\left(\mathrm{Y}_{3}\right)$ et le taux de pertes de turbidité $\left(\mathrm{Y}_{4}\right)$ (Tableau 5). Par ailleurs, aucune 
différence significative $(p>0,05)$ n'a été observée entre les valeurs expérimentales et les valeurs prédites (Tableau 5).

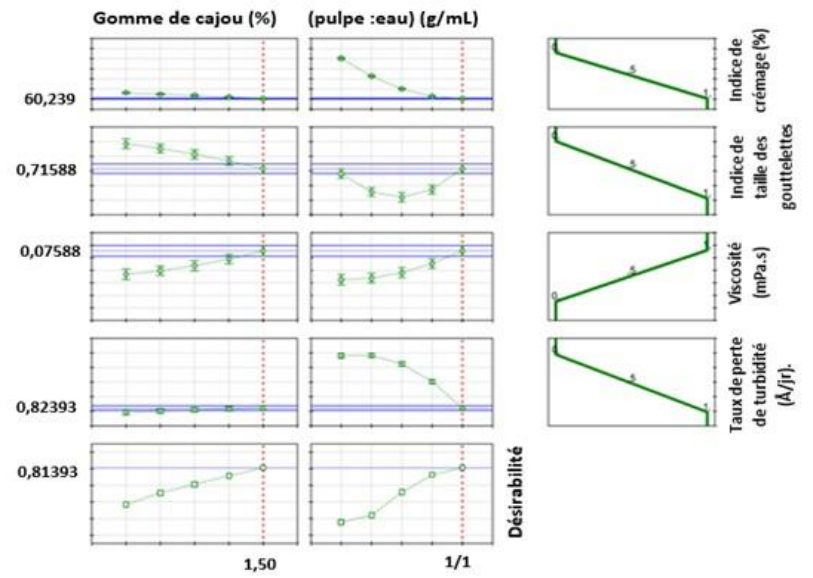

Figure 5. Profils des valeurs prévues et de désirabilité de l'indice de crémage $\left(\mathrm{Y}_{1}\right)$, l'indice de taille des gouttelettes $\left(\mathrm{Y}_{2}\right)$, de la viscosité $\left(\mathrm{Y}_{3}\right)$ et du taux de perte de turbidité $\left(\mathrm{Y}_{4}\right)$ du lait de coco.

Tableau 5. Valeurs prédites et expérimentales des réponses à des conditions optimales de variables indépendantes

\begin{tabular}{|l|l|l|l|l|}
\hline Variable & Niveaux \\
indépendantes & optimums & $\begin{array}{l}\text { Variables } \\
\text { dépendantes }\end{array}$ & \multicolumn{2}{|l|}{ Conditions optimales } \\
\cline { 3 - 5 } $\begin{array}{l}\text { Gomme de } \\
\text { cajou }\left(\mathrm{X}_{1}\right)\end{array}$ & $1,50 \%$ & $\begin{array}{l}\text { Indice de crémage } \\
\left(\mathrm{Y}_{1} ; \%\right)\end{array}$ & $60,30 \pm 0,09$ & 60,239 \\
\hline $\begin{array}{l}\text { Lait de coco } \\
\left(\mathrm{X}_{2}\right)\end{array}$ & $1 / 1(\mathrm{~g} / \mathrm{mL})$ & $\begin{array}{l}\text { Indice de taille des } \\
\text { gouttelettes }\left(\mathrm{Y}_{2}\right)\end{array}$ & $0,704 \pm 0,008$ & 0,715 \\
\hline & & $\begin{array}{l}\text { Viscosité } \\
\left(\mathrm{Y}_{3} ; \mathrm{mPa} . \mathrm{s}\right)\end{array}$ & $0,0761 \pm 0,0006$ & 0,0755 \\
\hline & $\begin{array}{l}\text { Taux de perte de } \\
\text { turbidité }\left(\mathrm{Y}_{4} ; \AA \text { À } / \mathrm{jr}\right)\end{array}$ & $0,821 \pm 0,016$ & 0,823 \\
\hline
\end{tabular}

a Moyenne \pm erreurs standard $(\mathrm{n}=3)$

\section{Conclusion}

$\mathrm{Au}$ terme de cette étude, nous retenons que l'optimisation par la méthodologie de surface de réponse (MSR), utilisant un plan factoriel complet 32 des constituants principaux du lait de coco peut être un outil précieux pour la production de lait de coco stabilisé par la gomme de cajou. Les modèles polynomiaux du second ordre, développés pour prédire les différentes caractéristiques du lait de coco, étaient très significatifs, avec des coefficients de détermination $R^{2}$ élevés $(>0,95)$, et des manques d'ajustement non significatifs $(p>0,05)$. Cette étude a montré que les caractéristiques des laits de coco étudiés étaient significativement influencées par la concentration en gomme de cajou et le ratio pulpes/eau. La formulation optimale pour produire un lait de coco avec un indice de crémage, un indice de taille des gouttelettes, 
une viscosité et un taux de perte de turbidité souhaitables a été fournie par le niveau combiné de $1,50 \%$ pour la gomme de cajou et de $1 / 1$ pour le ratio pulpes/eau. A cet égard, la gomme de cajou porterait un potentiel de valorisation dans l'industrie des boissons rafraichissantes.

\section{References:}

1. Abdullah, Z., Taip, F. S., Mustapa Kamal, S. M., \& Abdul Rahman, R. Z. (2018). Effect of sodium caseinate concentration and sonication amplitude on the stability and physical characteristics of homogenized coconut milk. Journal of Food Processing and Preservation, 42(11), e13773.

2. Arioui, F., Saada, D. A., \& Cheriguene, A. (2018). Functional properties of bovine bone gelatin and impact on physicochemical, microbiological and organoleptic quality of set yogurt. Biotechnology, 17(1), 1-11.

3. Baş, D., \& Boyac1, I. H. (2007). Modeling and optimization I: Usability of response surface methodology. Journal of food engineering, 78(3), 836-845.

4. Chang, H. W., Tan, T. B., Tan, P. Y., Abas, F., Lai, O. M., Wang, Y., Wang, Y., Imededdine, A. N., \& Tan, C. P. (2018). Physical properties and stability evaluation of fish oil-in-water emulsions stabilized using thiol-modified $\beta$-lactoglobulin fibrils-chitosan complex. Food research international, 105, 482-491.

5. Derringer, G., \& Suich, R. (1980). Simultaneous optimization of several response variables. Journal of quality technology, 12(4), 214219.

6. Ekanem, G. O., \& Ojimelukwe, P. C. (2017). Potentials of coconut milk as a substitute for cow milk in cheese making. Journal of Advances in Microbiology, 4(2), 1-9.

7. Fatimah, F., Gugule, S., \& Tallei, T. E. (2017). Characteristic of Coconut Milk powder made by variation of Coconut-Water Ratio, concentration of Tween and Guar gum. Journal of Applied Sciences Research, 13(6), 34-44.

8. Gharibzahedi, S. M. T., Mousavi, S. M., Hamedi, M., \& Ghasemlou, M. (2012). Response surface modeling for optimization of formulation variables and physical stability assessment of walnut oil-in-water beverage emulsions. Food Hydrocolloids, 26(1), 293-301.

9. Goralchuk, A., Grinchenko, O., Riabets O. \& Kotlyar, O. (2019). Food dispersion systems process stabilization. A review. Ukrainian Food Journal, 8(4), 699-732. 
10. Jiang, P., Xiang, D., \& Wang, X. (2016). Effect of different treatment on the properties of coconut milk emulsions. Food Science and Technology Research, 22(1), 83-89.

11. Jirapeangtong, K., Siriwatanayothin, S., \& Chiewchan, N. (2008). Effects of coconut sugar and stabilizing agents on stability and apparent viscosity of high-fat coconut milk. Journal of Food Engineering, 87(3), 422-427.

12. McClements, D. J. (2015). Food emulsions: principles, practices, and techniques. CRC press.

13. Mirhosseini, H., Tan, C. P., Hamid, N. S., \& Yusof, S. (2008). Effect of Arabic gum, xanthan gum and orange oil contents on $\zeta$-potential, conductivity, stability, size index and $\mathrm{pH}$ of orange beverage emulsion. Colloids and Surfaces A: Physicochemical and Engineering Aspects, 315(1-3), 47-56.

14. Montgomery, D. C. (2017). Design and analysis of experiments. John wiley \& sons.

15. Noorlaila, A., Siti Aziah, A., Asmeda, R., \& Norizzah, A. R. (2015). Emulsifying properties of extracted Okra (Abelmoschus esculentus L.) mucilage of different maturity index and its application in coconut milk emulsion. International Food Research Journal, 22(2), 782-787.

16. Onsaard, E., Vittayanont, M., Srigam, S., \& McClements, D. J. (2006). Comparison of properties of oil-in-water emulsions stabilized by coconut cream proteins with those stabilized by whey protein isolate. Food Research International, 39(1), 78-86.

17. Owens, C., Griffin, K., Khouryieh, H., \& Williams, K. (2018). Creaming and oxidative stability of fish oil-in-water emulsions stabilized by whey protein-xanthan-locust bean complexes: Impact of pH. Food Chemistry, 239, 314-322.

18. Peamprasart, T., \& Chiewchan, N. (2006). Effect of fat content and preheat treatment on the apparent viscosity of coconut milk after homogenization. Journal of Food Engineering, 77(3), 653-658.

19. Simuang, J., Chiewchan, N., \& Tansakul, A. (2004). Effects of fat content and temperature on the apparent viscosity of coconut milk. Journal of Food Engineering, 64(2), 193-197.

20. Tangsuphoom, N. \& Coupland, J. N. (2005). Effect of Heating and Homogenization on the Stability of Coconut Milk Emulsions. Journal of Food Science, 70(8), 466-470.

21. Tangsuphoom, N., \& Coupland, J. N. (2008). Effect of surface-active stabilizers on the microstructure and stability of coconut milk emulsions. Food Hydrocolloids, 22(7), 1233-1242. 